\title{
ANALYSIS OF PALMAR GRASP STRENGTH IN TERM NEWBORNS
}

\author{
J. Dionisio ${ }^{1}$, M.V. Moraes ${ }^{2}$, E. Tudella ${ }^{3}$, W.B. Carvalho ${ }^{1}$, V.L. Krebs ${ }^{1}$
}

${ }^{1}$ Paediatrics, University of São Paulo Medical School, São Paulo, ${ }^{2}$ Physiotherapy, Universidade Regional de Blumenau, Santa Catarina, ${ }^{3}$ Physiotherapy, Universidade Federal de São Carlos, São Carlos, Brazil

Background: The normal motor development includes an increase of the motor repertoire throughout life.

Aim: Analyze the palmar grasp strength in term newborns (TNB) during the first 72 hours after birth.

Methods: Cohort prospective study, in healthy TNB. Palmar grasp strength was assessed by a simple, noninvasive and painless procedure, with the equipment M-Flex ${ }^{\circledR}$. During the first 24-72 hours after birth, each newborn underwent a single assessment, consisting of hand width anthropometric measures and palmar grasp strength testing, where the equipment's rubber pad was placed at the base of fingers number 3,4 , and 5 for up to 30 seconds, performing three measurements for each hand. Assessed variables: age, time and maximum palmar grasp strength. ANOVA two-way parametric test was used considering $p<0.05$ as a significant value. The study was approved by the Ethics Committee.

Results: 216 newborns were included: 44 were 24 hours old, 97 were 48 hours old and 75 were 72 hours old; mean gestational age was 39 weeks $(\mathrm{SD}=1.47)$ and mean weight was $3167 \mathrm{~g}(\mathrm{SD}=462.9)$. The 72 hour-old newborns presented significantly higher palmar grasp strength when compared to 24 hour-old $(\mathrm{F}=0.2277$, $\mathrm{p}=0.01)$ and the 48 hour-old ones $(\mathrm{F}=0.06330, \mathrm{p}=0.01)$.

Conclusions: There was a significant increase in the palmar grasp strength among the TNB between 24 and 72 hours after birth, indicating the integrity and maturation of brain cortex. Palmar grasp strength measurement may constitute an important resource for early neonatal neurologic assessment.

Acknowledgments: We thank the Coordination for the Improvement of Higher Education Personnel (CAPES-Brazil). 